\title{
Worldwide increase in diabetes: implications for tuberculosis control
}

This article was published in the following Dove Press journal:

Research and Reports in Tropical Medicine

3 July 2014

Number of times this article has been viewed

\section{Susan P Fisher-Hoch \\ Division of Epidemiology, Human Genetics and Environmental Science, University of Texas School of Public Health, Brownsville Campus, Brownsville, TX, USA}

Video abstract

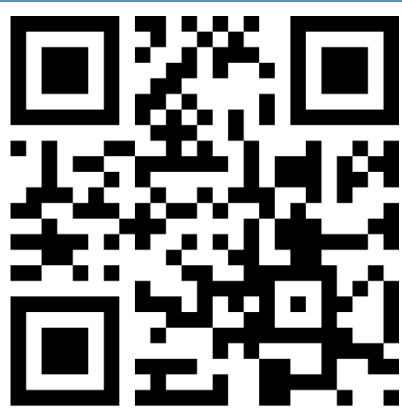

Point your SmartPhone at the code above. If you have a QR code reader the video abstract will appear. Or use: http://dvpr.es/ltT90 Ez
Correspondence: Susan P Fisher-Hoch Division of Epidemiology, Human Genetics and Environmental Science, University of Texas School of Public Health, 80 Fort Brown, Brownsville, Texas 78520, USA

Email susan.p.fisher-hoch@uth.tmc.edu
Abstract: Diabetes presents a greater threat to global tuberculosis (TB) control than previously appreciated, with risk of reversing the achievements of several decades. An estimated 382 million people worldwide currently have diabetes, half of whom are undiagnosed. Most live in low- and middle-income countries alongside many of the two billion individuals infected with TB. Though the frequency of TB in type 1 diabetes was known for centuries, only recently have we observed the tripling of TB in type 2 diabetes, most significantly in high-burden TB populations such as in Peru, Russia, and the People's Republic of China. In India diabetes is estimated to have increased TB cases by $46 \%$ between 1998 and 2008. Diabetes is a greater long-term threat to TB control than human immunodeficiency virus (HIV)/acquired immunodeficiency syndrome (AIDS) since ten-fold more people are affected by diabetes than HIV/ AIDS in larger geographic areas. Diabetes in TB increases drug resistance, treatment failure, and mortality, and may increase the spread of drug-resistant strains. Delayed or missed diagnosis fuels transmission of TB and hinders control of diabetes. Tailored treatment for diabetes patients requires well-designed clinical trials. The World Health Organization (WHO) framework for care and control of diabetes and TB needs improved screening strategies. Determination of how best to establish bi-directional screening is hampered by lack of affordable and reliable methods. Recommendations include education of health care providers, patients, and communities. Structured diabetes programs with registries and effective follow-up could be modeled on and communicate with existing TB programs. Vital research should address new diagnostic tools, lowering cost and evaluation of intervention strategies, as well as better understanding of the impaired immune responses that make diabetes patients more susceptible to TB leading to targeted therapies. Solutions will require the combination of good science, good decisionmaking, adequate funding, and political will.

Keywords: type 2 diabetes, MDR-TB, global public health

\section{Introduction}

In 1935 tuberculosis (TB) was listed among the four commonest causes of death in patients with diabetes mellitus. ${ }^{1}$ Indeed, before the introduction of insulin it was estimated that infections, most commonly TB, killed $20 \%$ of all diabetes patients. ${ }^{2}$ At that time many diabetes patients were young at onset and had type 1 diabetes, described as 'juvenile-onset diabetes'. Today type 1 has been eclipsed by type 2 diabetes, which is now responsible for over $90 \%$ of reported diabetes. Type 2 diabetes is a leading component with cardiovascular disease of the pandemic of noncommunicable diseases (NCDs) and is now increasingly seen in young people, including adolescents and children. ${ }^{3,4}$ Today it is estimated that 382 million people have diabetes, and this number is predicted to increase to 592 million by $2035 .{ }^{5}$ Type 2 diabetes and the NCDs 
to which it belongs can reasonably be listed as the pandemic of the 21 st century. ${ }^{4-6}$

Concurrently there are an estimated 2 billion individuals infected with TB in the course of another, well established and ancient pandemic. There were 8.6 million new active cases of TB in 2013, and 1.3 million deaths. ${ }^{7,8}$ In most countries effective programs reducing transmission by using directly observed therapy (DOTS) have been able to slow TB spread. ${ }^{9,10}$ However, the emergence of HIV/AIDS in the 1980s had major adverse impact on both the epidemiology and course of TB infections, adversely impacting the progress of TB control efforts. ${ }^{11}$ That the medical community was slow to recognize the importance of TB in HIV/AIDS increased the impact of the coinfection. ${ }^{12}$

Similarly, the insidious impact of diabetes on TB epidemiology, like that of AIDS in the early years, has been slowly recognized. Addressing the impact of diabetes on TB infections is complex. In addition to high rates of undiagnosed diabetes, those with diabetes have higher rates of undiagnosed TB. ${ }^{5,7}$ They are also thought to have more latent TB infections (LTBI), which may more frequently become active disease, possibly due to the effects of diabetes on the immune system. Management of comorbid diabetes and TB is challenging, with delayed mycobacterial clearance, high rates of relapse and deaths, and development of secondary drug resistance. In nearly all countries multidrug-resistant (MDR)-TB strains are spreading in the guise of a progressive secondary TB pandemic, threatening TB control programs. ${ }^{13,14}$ In countries with high rates of MDR-TB, diabetes has the potential to fuel primary spread of resistant strains. We have some understanding of the possible immune mechanisms through which diabetes creates susceptibility to TB but better knowledge will be critical in developing novel therapy to diminish the risk of TB to the diabetes patient.

The preceding discussion shows that diabetes presents profound threats to TB control, including MDR-TB. How to deal with these many threats is beginning to be addressed, but there are more questions than answers. Solutions will require the combination of good science, good decision-making, adequate funding, and political will. This review examines the advances and gaps in knowledge.

\section{Methods}

This review discusses the current knowledge of the impact of diabetes globally on the epidemiology of TB with particular emphasis on TB control, including MDR-TB. To do this a literature search was conducted using PubMed and Embase, supplemented by searches using Google Scholar to derive older citations and online reports, and by accessing the bibliographies of relevant literature, prominent among which are three formal systematic reviews with meta-analysis. ${ }^{15-17}$ Literature was reviewed from journals focused on the epidemiology of TB and diabetes, global public health, socioeconomic issues, immunology, and clinical and basic science. Ethical approval was not required for the purposes of this review.

\section{Results \\ Epidemiology of diabetes}

Diabetes was first named by Araetus of Cappadocia in the 2nd century AD after the Greek word for 'syphon' due to the characteristic polydipsia and polyuria, but was known to the ancient Egyptians at least 1,000 years earlier. ${ }^{18}$ The addition of mellitus to the scientific name came in the 18th century in Britain, when the sweet taste of urine in patients with diabetes was observed. The central involvement of the liver and the pancreas in glucose and insulin metabolism was first described in the 19th century. Much of the diabetes over the centuries would have been 'juvenile-onset' type 1 diabetes, an autoimmune disease, but the so-called 'maturity-onset' type 2 diabetes was certainly present from ancient times, most probable among the affluent in wealthier societies. Today the epidemiology of type 2 diabetes, driven by dramatic changes in lifestyles, has largely eclipsed that of type 1 diabetes. The misconception that type 2 diabetes is a disease only of the affluent elderly is also now fading. Eighty percent of people with diabetes now live in low- and middle-income countries (LMICs), and most are between 40 and 59 years of age. ${ }^{5}$ The changing lifestyles that accompany economic development have led to emerging 'hot-spots' for diabetes in the Middle East, Western Pacific, sub-Saharan Africa, and south-East Asia, where the disturbing trend toward onset at earlier ages is marked. Globally, roughly half of deaths due to diabetes are in those under 60 years of age, and in sub-Saharan Africa, 75\%. Of additional concern is that nearly half (46\%) of diabetes cases globally are undiagnosed, and in sub-Saharan Africa, this rises to $80 \%{ }^{5}$

Diabetes accounted for the deaths of 5.1 million people globally in 2013, similar in number to combined deaths from the major infectious diseases, including acquired immunodeficiency syndrome (AIDS), malaria, and TB. ${ }^{4}$ The People's Republic of China, India, the USA, and the Russian Federation are the most severely affected, but many countries have large numbers of cases including Brazil, several European countries, and even African countries (Nigeria, South Africa, and eastern African states). ${ }^{5}$ Many of these are countries with high-burden TB. 


\section{Epidemiology of TB}

The epidemiology of TB has an equally long history, having evolved over millennia rather than decades. TB has long been a major killer, only recently becoming controlled in high-income countries. Since the 1990s early case detection and effective control, specifically with DOTS, have been the mainstays of control, but improvement in living conditions and economic advancement were and still are a crucial element in reducing the burden. ${ }^{11}$ Despite the fact that in 2013 global prevalence and mortality from TB are in steady decline as a result of major international efforts, estimated annual incidence globally is only falling by about $2 \%$. The TB elimination target of 2050 (less than 1 case/million globally) requires a reduction of $20 \%$ annually - ten times the current rate. ${ }^{19}$ Several important factors limit the decline in incidence. First there is a large pool of undiagnosed cases who fuel transmission and are likely to continue to do so. Lack of resources is among the many reasons cases are missed. Currently TB REACH programs are attempting to address this gap by funding projects to detect increased numbers of cases earlier in disease. This program, funded by the Canadian International Development Agency, has so far resulted in a $33 \%$ increase in case detection overall. ${ }^{20}$ Secondly, there is also a large pool of people with LTBI, and detecting these cases and instigation of appropriate preventive treatment is difficult. Accurate tests for LTBI are expensive and unavailable in many places, and preventive medication has side effects that limit compliance. ${ }^{19}$ Finding these asymptomatic people with LTBI is also a logistic and costly challenge and difficult in high burden TB settings.

\section{MDR-TB}

The WHO describes MDR-TB as a public health crisis. ${ }^{21}$ Today an estimated 630,000 people globally are infected with MDR-TB, with a treatment failure rate of nearly $50 \%$, about 170,000 deaths annually, and many cases lost to follow-up. ${ }^{7}$ Only about $25 \%$ of the estimated MDR-TB cases are detected in high-burden countries. ${ }^{7}$ In Eastern Europe it is estimated that half previously treated patients have MDR-TB. ${ }^{21}$ In Belarus, one of the countries worst affected, one third of new cases were found to have MDR-TB, as did three quarters of previously treated patients. Over $10 \%$ of these patients had extensively drug-resistant TB (XDR-TB). ${ }^{22}$ The International Diabetes Federation estimates that Belarus had 445,250 diabetes cases in 2013. ${ }^{5}$ The reality is now that MDR-TB strains are spreading as primary infections in high-density populations. ${ }^{21}$

A major obstacle to control is that diagnosis of MDR-TB requires isolation of the organism or molecular testing, both of which have limited availability due to lack of resources and, where they are available, technical limitations. ${ }^{8}$ Indeed, the WHO estimates that less than $3 \%$ of patients with TB have had isolates tested for drug susceptibility. The WHO also estimates there are half a million incident cases of MDR-TB globally and 50,000 XDR-TB cases, accounting for about $3.6 \%$ of all TB. ${ }^{7}$ MDR-TB has the potential to become the dominant, potentially untreatable form of TB globally. ${ }^{8}$ The threat to all countries is now real, and requires commitment and leadership if MDR-TB is not to threaten global efforts to control TB. ${ }^{21}$ Better and less-expensive detection methods and new drugs are urgently needed.

\section{Diabetes and TB}

Though the menacing connection between diabetes and TB has been known for centuries it was largely forgotten following the introduction of insulin for the treatment of type 1 diabetes in the 1930s, and thereafter the introduction of streptomycin for TB. ${ }^{23}$ It began to re-emerge only in the $1980 \mathrm{~s}$ and 90s with a few reports, largely overlooked, from Chile, Tanzania, and Mexico. ${ }^{24-26} \mathrm{~A}$ larger study in Mexico in 2004, and our own study across the US/Mexico border in 2007, confirmed the importance of the association in Mexicans and Mexican Americans. ${ }^{27,28}$ At this point the severity of the global challenge became recognized, with reports of a threefold increase in active TB associated with diabetes. ${ }^{17}$

Diabetes and the acquired immunodeficiency syndrome (HIV/AIDS) both increase susceptibility to TB. The risk of TB in HIV-infected persons is now well documented. HIV/ AIDS has had a major, adverse impact on TB control and on the spread of MDR-TB, and more recently XDR-TB..$^{19,29}$ After decades of emphasis in global health on infectious diseases, it is only now becoming recognized that NCDs, including diabetes, are of equal importance globally, and even more so than infectious diseases in terms of total morbidity and mortality in most communities. This is significantly so in LMICs, many of which are high-burden TB countries. ${ }^{4}$

The insidious impact of diabetes on TB epidemiology, mirroring that of HIV/AIDS in the early years, has been only slowly recognized. ${ }^{12}$ Alcohol dependence and smoking have long been recognized as risk factors for active TB, but type 2 diabetes is the newcomer to the NCD risk factor list and is likely the new elephant in the room. Though, as previously stated, the association of TB with type 2 diabetes first emerged in the late 1980s and 1990s in isolated reports from places as far apart as Chile and Tanzania, these remained buried in the literature. ${ }^{24,25,30}$ The real threat of diabetes to TB control became recognized as serious, albeit slowly, only in the first decade of the 21 st century. The delay was 
increased in part by resistance from some major agencies where the focus on HIV/AIDS and social risk factors was dominant. $^{31}$

Thus in the first decade of the 21 st century it became clear, first in Mexicans and Mexican Americans, that the increased odds ratio for TB in diabetes was as high as threeto six-fold. ${ }^{26-28,32,33}$ An observation in these studies was that TB patients with diabetes had an entirely different risk-factor profile from those without diabetes. ${ }^{28}$ Patients with TB and diabetes had none of the well-recognized medical and social risk factors such as HIV/AIDS infection, incarceration, and alcohol or drug addiction. In one study, diabetes patients with TB were generally older than those without diabetes. ${ }^{28}$ Indeed a diabetes patient with TB may be a middle-aged citizen whose exposure may have simply been a visit to a crowded market.

Reports from across the globe followed rapidly. ${ }^{17,34-37}$ The impact in the People's Republic of China and in India is particularly grave. ${ }^{12}$ Reports from centers in India using glycosylated hemoglobin $\left(\mathrm{HbA}_{1 \mathrm{c}}\right)$ as a diagnostic tool showed that nearly half of the TB patients seen in clinics in some states had diabetes as a risk factor, and only half of these knew they had diabetes. ${ }^{36,38}$ Poorly controlled diabetes increases the risk of TB a further two-fold. ${ }^{35}$ In our own studies and those of others even prediabetes increases the risk of TB. ${ }^{36}$ Today the re-emergence of the association of diabetes with TB with the pandemic of type 2 diabetes is widely established, with measures for control recommended and in some cases implemented. ${ }^{12,16,17,39}$ The interface of these two important diseases was solidly documented for the first time in 2009 with a comprehensive meta-analysis of the literature published along with an assessment of the global situation and determination of the research agenda. ${ }^{17,31}$ This led to the WHO framework for care and control of diabetes and TB published in 2011. ${ }^{40}$ This document recommends joint coordination between diabetes and TB programs within countries; surveillance for TB in diabetes patients, and diabetes in TB patients; and development by national programs of core sets of indicators and tools, including adaptation of DOTS systems for diabetes surveillance, reporting, and control. Further predictions of the impact of diabetes on TB were now assessed and the threat of diabetes to TB control globally fully articulated. ${ }^{12,39,41-45}$ The impact is recognized now to be greatest in high-burden TB countries such as Peru and the Russian Federation, and in the People's Republic of China and in India where diabetes is estimated to have increased the number of TB cases by $46 \%$ between 1998 and 2008 (Table 1). ${ }^{38,46,47}$
The early failure to appreciate the impact of HIV/AIDS on TB was reviewed by Harries et al as a warning of the consequences of failure to act promptly on the threat of diabetes. ${ }^{12}$ For example, in our Hispanic population on the US/Mexico border, where HIV/AIDS is not common, the individual attributable fraction for HIV/AIDS is $94 \%$, and for diabetes $68 \%$, but the population attributable fraction (PAF) for HIV/AIDS is only $3 \%$ compared with $24 \%$ for diabetes. ${ }^{33}$ Indeed, in Europe today NCDs, including diabetes, are more important drivers of TB than HIV/AIDS. ${ }^{48}$ Observations in Central Europe are also striking, with a PAF for diabetes of $14.7 \%$ compared with only $2.9 \%$ for HIV/AIDS. Overall in established market economies the PAF for diabetes in TB is $14.4 \%$ compared with $6.4 \%$ for AIDS. This makes diabetes the second most important risk factor for TB in these regions, second only to smoking in magnitude. ${ }^{48}$ The increase in prevalence of diabetes, however, is most significant in the LMICs, particularly in south-East Asia, the Western Pacific and Africa. Seventy-nine percent of all diabetes and $95 \%$ of all TB is in LMICs. ${ }^{41}$ People with diabetes in poorer countries are more likely than those in wealthier countries to have TB and the projected increases in diabetes prevalence in these places is therefore likely to have major impact on the spread of TB. ${ }^{44}$ In some of these regions MDR-TB rates are very high, and here primary spread of MDR-TB among diabetes patients is set to become a significant threat to MDR-TB control.

LTBI is particularly important in the context of diabetes, since we have evidence using genotyping of isolates that half of the active TB infections associated with diabetes are likely due to reactivated $\mathrm{TB}^{27}$ The age distribution in this study in Veracruz, Mexico, showed that the age of the reactivated diabetes patients was older, with the majority, $78 / 130$ (60\%), in the 45-64 age range, compared with 15/42 (35\%) in the same age range among the clustered cases who were mostly younger. ${ }^{27}$ Screening for LTBI among diabetes patients, particularly in those at high risk for exposure to TB, should be seriously considered. Such individuals should be primarily sought in countries with high TB transmission or, in the case of more developed countries, in those emigrating from high-burden TB countries or communities. Further studies using interferon-gamma release (IGRA) assays and isolate genotyping are needed to clarify the risk presented by LTBI in diabetes patients in a range of geographic and social settings.

It is now generally established that diabetes is an individual risk factor for active $\mathrm{TB}$, with an approximate threefold greater risk. This risk is reviewed in a comprehensive 
systematic review with meta-analysis. ${ }^{44}$ While the risk of TB in an exposed individual with HIV infection is greater than for someone with diabetes, the threat of diabetes to TB control is even greater at the population level than HIV/AIDS because the global burden of diabetes is orders of magnitude greater. UNAIDS reports that in 2012 there were 35.3 million people living with HIV/AIDS, and TB remains their leading cause of death. ${ }^{49}$ That is less than $10 \%$ of the number (382 million) living with diabetes. ${ }^{5}$ These numbers firmly establish the risk of diabetes to TB control globally.

\section{Pathophysiology of susceptibility to TB in diabetes}

Understanding how diabetes predisposes to active TB is central to developing new therapy both to protect diabetes patients and to improve TB treatment outcomes. Certainly, preventing or controlling diabetes is the simplest approach, but notoriously difficult to achieve, particularly when half the patients are unaware of their condition. The chronic inflammatory syndrome characteristic of diabetes affects both the innate and adaptive immune systems, many components of which are persistently and significantly upregulated. Though immune responses to infectious antigens are brisk in diabetes, we are beginning to understand that their effectiveness in controlling infections is conversely impaired. ${ }^{50}$

Innate and acquired immune responses are not separate entities but operate in concert. Complex defects in both arms of the immune response to infectious agents, including TB, in diabetes are known to involve antibody response, neutrophil and macrophage function, CD4+/CD8 ratios, and T-cell functions. ${ }^{51}$ There are several neutrophil defects characteristic of diabetes that may be critical in TB. Though adherence of neutrophils to vascular endothelium is increased, chemotaxis and transmigration into tissue are reduced. ${ }^{52}$ There are also impairments in neutrophil migration, ${ }^{53}$ phagocytosis,${ }^{54}$ production of reactive oxygen species, ${ }^{55}$ and apoptosis. ${ }^{56}$ These defects result in decreased ability to respond to sites of infection; inability to clear pathogens; and excessive, damaging inflammation. ${ }^{57,58}$ Macrophages and monocytes also have impairments in critical defense mechanisms in TB infection including phagocytosis, activation, and antigen presentation. ${ }^{34}$ Alveolar macrophages have been shown to have reduced antimicrobial activity in response to challenge with Mycobacterium tuberculosis. ${ }^{59}$ Also reported is dysregulation of adhesion molecules E-selectin, vascular adhesion molecule-1, and intracellular adhesion molecule-1, all of which are upregulated during the innate immune response to aid in the recruitment of macrophages and other leukocytes to sites of infection. ${ }^{60}$

To understand the processes it is important to view diabetes as a proinflammatory state with elevated cytokines, including those normally involved in control of M. tuberculosis. Despite significantly higher innate and type 1 cytokine responses such as interferon gamma, important in control of M. tuberculosis, diabetes patients fail to control TB adequately. ${ }^{61}$ This failure suggests some defect in pathways downstream from key cytokines. This hypothesis is supported by evidence that stimulation of whole blood from diabetes patients with heat-killed pneumococci results in a ten-fold increase in the secretion of interferon gamma, interleukin (IL)-6, and IL-17 compared with blood from normal controls; nevertheless antibody production and killing of pneumococci is impaired. Furthermore, CD4+ and T-helper 17 (Th-17) cell memory responses are impaired in diabetes and we have recently also found that neutrophils from diabetes patients producing neutrophil extracellular traps when exposed to Streptococcus pneumoniae exhibited impaired ability to kill bacteria. ${ }^{62}$ Another emerging hypothesis is that the elevated levels in diabetes of cytokines IL-6 and IL-1 $\beta$ (which promote Th-17 cell differentiation) skews the balance of T-cells to produce higher levels of Th-17 cells and suppresses the levels of inflammation mediating regulatory T-cells. ${ }^{63,64}$ Given the impairments that diabetes delivers to a whole range of poorly understood immune responses critical in $\mathrm{TB}$, considerably more basic research is needed before we can have a sufficiently clear picture to design and develop targeted therapies. Taken as a whole the current literature suggests that a chronic proinflammatory state in diabetes while exhibiting hyper-responsiveness to invading organisms such as $M$. tuberculosis reduces ability to deliver the final blow and kill the invader.

\section{Impact of diabetes on TB treatment and outcomes}

Diabetes impairs responses to $M$. tuberculosis in multiple ways. Obesity compounds the problems presented by diabetes since obesity of itself is a risk factor for many pulmonary infections ${ }^{50}$ The catabolic effects of the infection destabilize blood glucose levels. Rifampicin (an antimycobacterial drug) itself may induce hyperglycemia, and the effects on liver metabolism of this drug may result in decreased efficacy of antidiabetes medication. Isoniazid may worsen peripheral neuropathy. In general, diabetes patients do not tolerate antituberculosis therapy well and diabetes management 
is compromised, resulting in complications and reduced compliance. $^{41}$

Possibly as a result of problems with treatment but also consequent to the increased mycobacterial load observed in diabetes, diabetes patients with TB take longer to clear the organisms from sputum. ${ }^{15,65}$ Diabetes also increases the likelihood of drug resistance (including XDR-TB) and TB mortality. ${ }^{15,66,67}$ It is also now clear that diabetes also increases relative risk of treatment failure and/or death in $\mathrm{TB}$, as reported in another systematic review with metaanalysis, by 1.69 (95\% confidence interval 1.36, 2.12). ${ }^{15}$ One study reported an effect estimate for death of 4.8 in TB with diabetes (95\% confidence interval $2.0,11.6)$. The fear is now that in high-burden TB countries with high rates of MDR-TB, diabetes will fuel transmission of drug-resistant strains. These challenges together and singly suggest that longer treatment schedules tailored to use in diabetes may be needed if not essential. This will require well-designed clinical trials with results guiding affordable and effective decisions.

\section{Bidirectional screening for diabetes and TB: strategies and obstacles}

The preceding discussion shows that diabetes presents a profound challenge to TB control, including MDR-TB. So, what is to be done? In recent years efforts of the WHO and others have resulted in significant declines in prevalence and death rates from TB. This success is, however, mirrored by a lesser decline in the incidence of new cases - far short of the decline needed for elimination of the disease. ${ }^{19}$ Two major impediments to control are missed or delayed diagnosis of active cases that fuel transmission and the large pool of latently infected people with LTBI who later develop active disease. Both are prominent in patients with diabetes. As also discussed previously, data from Mexico suggest that about half the cases of diabetes patients with TB result from reactivation of LTBI, with the remainder being new infections. These conclusions are based on sequencing data showing clustering of new infections with the same genotype, and sporadic appearance of what are presumed to be reactivation infections with a wide range of genotypes. ${ }^{27}$ We need new studies to determine the geographic distribution of reactivation and new transmission in diabetes.

As long ago as 1974 the WHO advised against 'indiscriminate' mass screening for TB. ${ }^{19}$ However, this advice was based on populations with low TB burdens. In the high-burden countries, resources for diagnosis and treatment were in any event inadequate to draw any conclusions about the cost-effectiveness. Screening of some high-risk groups, specifically people with HIV/AIDS and contacts of cases, has continued, but diabetes was only recognized as a risk factor warranting screening in $2011 .{ }^{40}$ Currently the WHO is reconsidering screening advice, but in the meantime, the recognition of the association of these two pandemics means that novel strategies for screening need to be developed to address the new risks, and that this is particularly urgent in high-burden countries for both diseases.

Two approaches are obvious from the outset: i) screening for diabetes in TB patients, and ii) screening for TB in diabetes patients. Current data on both approaches have also been systematically reviewed with meta-analysis. ${ }^{16}$ There are many challenges. Screening for diabetes in TB clinics was recommended in the WHO framework and is being field tested in India, Mexico, and the People's Republic of China. ${ }^{68}$ Many diabetes patients are unaware of their condition so do not self-report, particularly in LMICs, where funds and facilities for diabetes diagnosis and management are frequently lacking. Diabetes rates among TB patients ranging from about $13 \%$ to $50 \%$ and even higher have been reported in a systematic review with meta-analysis. ${ }^{17}$

To be useful for case management, diagnosis of diabetes needs to be made at the time of diagnosis of TB. However, methods of screening for diabetes are problematic and correlate imperfectly. ${ }^{69}$ Fasting blood glucose using strips is a simple and cheap test for diabetes that is widely used, but insensitive. It requires an already sick patient to be fasting. Random blood glucose can be used, and if this is over $200 \mathrm{mg} / \mathrm{dL}$ it is a reasonable and cheap indicator of potential diabetes but needs confirmation. An oral glucose tolerance test in acutely sick patients with TB has been used successfully, but it is expensive and is similarly not desirable since it requires fasting, an oral glucose challenge, and blood glucose monitoring over 2 hours in a sick and probably infectious patient. The oral glucose tolerance test commonly identifies more patients with potential diabetes than other methods. ${ }^{24,69}$ Regardless, TB interferes with glucose control so that results from all these assays can be misleading, and in any event they do not correlate well when directly compared. An optimal solution is to measure $\mathrm{HbA}_{1 \mathrm{c}}$, which does not require fasting and which also circumvents the issue of the effects of the acute infection on glucose control, since it is a measure of average blood glucose over the preceding 3 months. Commercial pointof-care assays for $\mathrm{HbA}_{1 \mathrm{c}}$ are now available requiring small volumes of blood and providing immediate results, but the reproducibility of some of these assays is in question. ${ }^{70}$ 
Whatever assay is used, it is expensive and thus out of reach for most LMIC countries. However, the desirability of determining diabetes status at the time of TB diagnosis in a single visit is considerable, since the knowledge will affect immediate clinical decisions about treatment and follow-up of the patient in addition to the management of diabetes and counseling of the patient. Introduction of treatment regimens designed for diabetes necessitates diagnosis at the time of presentation of the patient with TB. Wide availability of a rapid, reproducible, accurate but inexpensive assay is critical.

Diabetes patients clearly contribute to the large pool of undiagnosed TB worldwide and will do so increasingly as the pandemic progresses. ${ }^{16,71}$ Screening for undiagnosed active TB in diabetes is problematic, with even more unanswered questions. Nevertheless, it can be successfully done as has been shown by recent clinical studies. In high-burden TB areas the yield of TB diagnoses in diabetes clinics can be as much as three- to seven-fold higher than in the general population. ${ }^{16,71}$ In these settings, where screening is most needed, resources are again often lacking. Diagnosis of TB requires expertise outside the training and skills of the average diabetes clinic, such as applying and reading tuberculin skin tests, taking adequate sputum specimens and handling them safely, and reading of both smears and X-rays. Furthermore, all these tests are insensitive.

Even more challenging is screening for LTBI in diabetes so that treatment may be used to prevent open infection. The size of the pool of LTBI in diabetes patients is unknown, but is likely to be large, and certainly presents further opportunities for spread if these people progress to active infection. A major problem is that rates of conversion of LTBI to TB are unknown in diabetes, and accurate data to drive decisionmaking about screening will be difficult and expensive to obtain, requiring longitudinal studies.

Again, the most sensitive and specific assays, such as the IGRAs, are expensive, technically quite demanding (they require an incubation step and experienced reading) and thus out of reach in most LMIC settings. ${ }^{7}$ The WHO also recommends that IGRAs be used "in conjunction with risk assessment, radiography and other medical and diagnostic indications". "Evaluation has mostly been in high-income countries, and there are insufficient data to assess the use of IGRAs in LMICs, particularly in high-burden countries or those with high rates of HIV/AIDS. Most diabetes clinics will not have any of these capabilities on site, and moving a patient with diabetes to a TB clinic for diagnosis may add risk of further exposure, including exposure to MDR-TB strains. ${ }^{7}$ The risk of misdiagnosis and either unnecessary, or lack of, treatment is high in these circumstances. For these many reasons the WHO has not issued recommendations for the use of IGRAs in LMICs. ${ }^{7}$

Simple screening questions for active TB in diabetes have been developed and tested, but their sensitivity and specificity are poor. ${ }^{12,71}$ Even with a simple screening tool in place, we return to the issue of confirmation of the diagnosis. One potential solution that needs to be tested in high-burden TB populations is combining a simple questionnaire with the use of molecular diagnostic tools. This simple technique can be placed and operated in diabetes clinics and has the added advantage of identification of rifampicin-resistant strains and thus presumption of potential MDR-TB infections. However, these assays require collection of a sputum specimen, and thus a productive cough. Confirmation by isolation and complete sensitivity testing is also highly desirable. Diabetes patients living in high-burden settings will need repeated screenings. Poor record keeping and documentation in diabetes services and lack of established follow-up procedures for diabetic patients are further obstacles to implementation of effective programs.

In anticipation of improvements in diagnostic tools for both TB and diabetes there needs to be more research aimed at evaluating strategies and lowering costs. A simpler and less controversial approach that could be more immediately implemented is the education of diabetes care personnel, and indeed diabetes patients, on the risks, signs, and symptoms of TB. This would increase the possibility that potential cases be identified early and referred to diagnostic facilities. However, new approaches to education will need to be evaluated in different socioeconomic and cultural settings since the patients themselves may be mostly uneducated and uninsured, often unemployed, and from backgrounds with mixed beliefs affecting health-seeking behaviors. Furthermore, out-of-pocket expenses for management of type 2 diabetes are a common obstacle to case management. Connecting and modeling the diabetes clinic programs, which in most cases are poorly and inconsistently structured, with the better-structured TB clinics trained to deliver DOTS, will be important. Exchange of information between these primary care providers and shared data and training is needed at the country level. International experts in diabetes observe that the diabetes clinics in most countries would greatly benefit from the organization and structures of the TB programs that already exist, based on case detection and outcome measurements. ${ }^{72}$ Much could be gained by developing better overall diabetes clinic structures - specifically, consistent 
protocols, procedures, and documentation - and linking them with TB programs within each country. ${ }^{73}$

\section{Discussion}

The diabetes pandemic presents a much bigger threat to control of TB including MDR-TB than previously appreciated. The sheer numbers of at-risk people in high-burden TB countries is set to drive annual TB incidence for years to come unless action is taken. Diabetes patients with TB have high mycobacillary burdens at diagnosis, which they clear more slowly; higher rates of relapse; and higher rates of MDR-TB. They are also more likely to die of their infection.

A striking finding has been that the diabetes patient with TB is likely to be older in some circumstances, a woman, and without any of the sociopathic risk factors frequently associated with TB. This is shown to be the case in relatively developed urban cities found on the US/Mexico border where exposure can occur in everyday settings, such as a crowded, ill-ventilated department store, market, or café. With increased populations globally affected by diabetes, especially in the crowded megacities, diabetes in ordinary market goers, regular citizens going about their daily business, is likely to become a very common mode of spread of TB.

Given the diagnostic and cultural challenges in many high-burden countries, no quick fixes are currently available. However, many efforts are moving us toward understanding what steps need to be taken. Research aimed at evaluating diagnostic and treatment strategies to identify patients with diabetes and TB in LMICs are yielding useful information, including surprising numbers showing just how many people are at risk and how widespread the problem is. Studies are also beginning to define what strategies could be effective in detecting early TB in diabetes, and identifying TB patients at elevated risk of poor outcomes from concurrent diabetes. Proposals to structure diabetes care with registries and effective follow-up modeled on existing TB programs are wise, and if resources are made available, achievable. Education of health care providers, patients, and communities is a useful and well-tried tool, though culture-specific programs need to be developed and evaluated, and then applied on a large scale. Cohort monitoring of diabetes patients has been recommended, but for this and other initiatives, the will to provide resources is again needed.

Finally, the increased prevalence of diabetes has the potential to spread MDR-TB. These drug-resistant strains already threaten to become the dominant, potentially less treatable form of TB globally. ${ }^{8}$ Documentation shows that diabetes patients have higher rates of relapse and drug resistance than those without diabetes. Diabetes therefore has the potential to increase the number of resistant strains, and to spread them easily in everyday settings in LMICs. Diabetes will therefore be a considerable risk to control of MDR-TB.

The threat of this emerging interface of NCDs and infections is new and large. Unless we take steps to mitigate the effects globally, the achievements of several decades of TB control could be quickly negated.

\section{Disclosure}

The author reports no conflicts of interest in this work.

\section{References}

1. Flynn JM. The changing cause of death in diabetes mellitus. Am J Med Sci. 1935;189:157.

2. Bell J, Hockaday T. Diabetes mellitus. In: Wetherall DJ, Ledingham JGG, Warrell DA, editors. Oxford Textbook of Medicine. 3rd ed. Oxford: Oxford University Press; 1996;1448-1504.

3. cdc.gov [homepage on the Internet]. National diabetes prevention program. Centers for Disease Control and Prevention; 2014 [updated January 13, 2014]. Available from: http://www.cdc.gov/diabetes/prevention/. Accessed March 20, 2014.

4. World Health Organization. Global Status Report on Noncommunicable Diseases 2010. Geneva: World Health Organization; 2011. Available from: http://www.who.int/nmh/publications/ncd_report2010/en/. Accessed March 20, 2014.

5. International Diabetes Federation. IDF Diabetes Atlas, Sixth Edition. Brussels: International Diabetes Federation; 2013. Available from: http:// www.idf.org/diabetesatlas/download-book. Accessed March 20, 2014.

6. Fisher-Hoch SP. Diabetes and tuberculosis: a twenty-first century plague? Int J Tuberc Lung Dis. 2011;15(11):1422.

7. World Health Organization. Tuberculosis IGRA TB tests: Policy statement 2011: the use of TB Interferon-Gamma Release Assays (IGRAs) in Low- and Middle-income Countries. 2014. Available from: http:// www.who.int/tb/features_archive/igra_factsheet_oct2011.pdf. Accessed April 30, 2014.

8. Lynch JB. Multidrug-resistant Tuberculosis. Med Clin North Am. 2013;97(4):553-579.

9. Raviglione M, Marais B, Floyd K, et al. Scaling up interventions to achieve global tuberculosis control: progress and new developments. Lancet. 2012;379(9829):1902-1913.

10. Dye C, Lönnroth K, Jaramillo E, Williams BG, Raviglione M. Trends in tuberculosis incidence and their determinants in 134 countries. Bull World Health Organ. 2009;87(9):683-691.

11. Lönnroth K, Jaramillo E, Williams BG, Dye C, Raviglione M. Drivers of tuberculosis epidemics: the role of risk factors and social determinants. Soc Sci Med. 2009;68(12):2240-2246.

12. Harries AD, Lin Y, Satyanarayana S, et al. The looming epidemic of diabetes-associated tuberculosis: learning lessons from HIV-associated tuberculosis. Int J Tuberc Lung Dis. 2011;15(11):1436-1444.

13. Shah NS, Wright A, Bai GH, et al. Worldwide emergence of extensively drug-resistant tuberculosis. Emerg Infect Dis. 2007;13(3):380-387.

14. Cegielski JP. Extensively drug-resistant tuberculosis: "there must be some kind of way out of here". Clin Infect Dis. 2010;50(Suppl 3): S195-S200

15. Baker MA, Harries AD, Jeon CY, et al. The impact of diabetes on tuberculosis treatment outcomes: a systematic review. BMC Med. 2011;9:81.

16. Jeon CY, Harries AD, Baker MA, et al. Bi-directional screening for tuberculosis and diabetes: a systematic review. Trop Med Int Health. 2010;15(11):1300-1314. 
17. Jeon CY, Murray MB. Diabetes mellitus increases the risk of active tuberculosis: a systematic review of 13 observational studies. PLoS Med. 2008;5(7):e152.

18. Ahmed AM. History of diabetes mellitus. Saudi Med J. 2002;23: 373-378.

19. Lönnroth K, Corbett E, Golub J, et al. Systematic screening for active tuberculosis: rationale, definitions and key considerations. Int J Tuberc Lung Dis. 2013;17(3):289-298.

20. stopTB.org [homepage on the Internet]. TB REACH - results. Stop TB Partnership; 2014. Available from: http://www.stoptb.org/global/ awards/tbreach/achievements.asp. Accessed March 20, 2014.

21. Abubakar I, Zignol M, Falzon D, et al. Drug-resistant tuberculosis: time for visionary political leadership. Lancet Infect Dis. 2013;13(6): 529-539.

22. Skrahina A, Hurevich H, Zalutskaya A, et al. Multidrug-resistant tuberculosis in Belarus: the size of the problem and associated risk factors Bull World Health Organ. 2013;91(1):36-45.

23. Oscarsson N, Silwer H. Incidence of pulmonary tuberculosis among diabetics. Acta Med Scand. 1958;160(Suppl 335):23-48.

24. Mugusi F, Swai AB, Alberti KG, McLarty DG. Increased prevalence of diabetes mellitus in patients with pulmonary tuberculosis in Tanzania Tubercle. 1990;71(4):271-276.

25. Olmos P, Donoso J, Rojas N, et al. [Tuberculosis and diabetes mellitus: a longitudinal-retrospective study in a teaching hospital]. Rev Med Chil. 1989;117(9):979-983. Spanish [with English abstract].

26. Pablos-Méndez A, Blustein J, Knirsch CA. The role of diabetes mellitus in the higher prevalence of tuberculosis among Hispanics. Am J Public Health. 1997;87(4):574-579.

27. Ponce-De-Leon A, Garcia-Garcia Md Mde L, Garcia-Sancho MC, et al. Tuberculosis and diabetes in southern Mexico. Diabetes Care. 2004;27(7):1584-1590.

28. Restrepo BI, Fisher-Hoch SP, Crespo JG, et al. Type 2 diabetes and tuberculosis in a dynamic bi-national border population. Epidemiol Infect. 2007;135(30):483-491.

29. World Health Organization. Report of the Second Meeting of the WHO Task Force on XDR-TB, WHO Headquarters, Geneva, Switzerland, April 9-10, 2008. Geneva, Switzerland: World Health Organization; 2008.

30. Swai AB, McLarty DG, Mugusi F. Tuberculosis in diabetic patients in Tanzania. Trop Doct. 1990;20(4):147-150.

31. Harries AD, Murray MB, Jeon CY, et al. Defining the research agenda to reduce the joint burden of disease from diabetes mellitus and tuberculosis. Trop Med Int Health. 2010;15(6):659-663.

32. Pérez A, Brown HS 3rd, Restrepo BI. Association between tuberculosis and diabetes in the Mexican border and non-border regions of Texas. Am J Trop Med Hyg. 2006;74(4):604-611.

33. Restrepo BI, Camerlin AJ, Rahbar MH, et al. Cross-sectional assessment reveals high diabetes prevalence among newly-diagnosed tuberculosis cases. Bull World Health Organ. 2011;89(5):352-359.

34. Dooley KE, Chaisson RE. Tuberculosis and diabetes mellitus: convergence of two epidemics. Lancet Infect Dis. 2009;9(12):737-746.

35. Leung CC, Lam TH, Chan WM, et al. Diabetic control and risk of tuberculosis: a cohort study. Am J Epidemiol. 2008;167(12):1486-1494.

36. Viswanathan V, Kumpatla S, Aravindalochanan V, et al. Prevalence of diabetes and pre-diabetes and associated risk factors among tuberculosis patients in India. PLoS One. 2012;7(7):e41367.

37. Alisjahbana B, Van Crevel R, Sahiratmadja E, et al. Diabetes mellitus is strongly associated with tuberculosis in Indonesia. Int JTuberc Lung Dis. 2006;10(6):696-700.

38. Balakrishnan S, Vijayan S, Nair S, et al. High diabetes prevalence among tuberculosis cases in Kerala, India. PLoS One. 2012;7(10):e46502.

39. Young F, Critchley JA, Johnstone LK, Unwin NC. A review of comorbidity between infectious and chronic disease in Sub Saharan Africa: TB and diabetes mellitus, HIV and metabolic syndrome, and the impact of globalization. Global Health. 2009;5:9.

40. International Union Against Tuberculosis and Lung Disease; World Health Organization. Collaborative Framework for Care and Control of Tuberculosis and Diabetes. Geneva: World Health Organization; 2011. Available from: http://www.who.int/tb/features_archive/diabetes_link/ en/. Accessed March 20, 2014.
41. Kapur A, Harries AD. The double burden of diabetes and tuberculosis - public health implications. Diabetes Res Clin Pract. 2013;101(1):10-19.

42. Hall V, Thomsen RW, Henriksen O, Lohse N. Diabetes in Sub Saharan Africa 1999-2011: epidemiology and public health implications. A systematic review. BMC Public Health. 2011;11:564.

43. Dye C, Williams BG. The population dynamics and control of tuberculosis. Science. 2010;328(5980):856-861.

44. Goldhaber-Fiebert JD, Jeon CY, Cohen T, Murray MB. Diabetes mellitus and tuberculosis in countries with high tuberculosis burdens: individual risks and social determinants. Int J Epidemiol. 2011;40(2):417-428.

45. Ruslami R, Aarnoutse RE, Alisjahbana B, van der Ven AJ, Van Crevel R. Implications of the global increase of diabetes for tuberculosis control and patient care. Trop Med Int Health. 2010;15(11):1289-1299.

46. Dye C, Bourdin Trunz B, Lönnroth K, Roglic G, Williams BG. Nutrition, diabetes and tuberculosis in the epidemiological transition. PLoS One. 2011;6(6):e21161.

47. Bygbjerg IC. Double burden of noncommunicable and infectious diseases in developing countries. Science. 2012;337(6101):1499-1501.

48. Creswell J, Raviglione M, Ottmani S, et al. Tuberculosis and noncommunicable diseases: neglected links and missed opportunities. Eur Respir J. 2011;37(5):1269-1282.

49. UNAIDS. 2013 Global Fact Sheet. Geneva: UNAIDS; 2013. Available from: http://www.unaids.org/en/Resources/PressCentre/Factsheets/. Accessed March 20, 2014.

50. Fisher-Hoch SP, Mathews CE, McCormick JB. Obesity, diabetes and pneumonia: the menacing interface of non-communicable and infectious diseases. Trop Med Int Health. 2013;18(12):1510-1519.

51. Pickup JC. Inflammation and activated innate immunity in the pathogenesis of type 2 diabetes. Diabetes Care. 2004;27(3):813-823.

52. Peleg AY, Weerarathna T, McCarthy JS, Davis TM. Common infections in diabetes: pathogenesis, management and relationship to glycaemic control. Diabetes Metab Res Rev. 2007;23(1):3-13.

53. Sawant JM. Biochemical changes in polymorphonuclear leucocytes in diabetic patients. J Postgrad Med. 1993;39(4):183-186.

54. Krol E, Agueel R, Banue S, Smogorzewski M, Kumar D, Massry SG. Amlodipine reverses the elevation in $[\mathrm{Ca} 2+] \mathrm{i}$ and the impairment of phagocytosis in PMNLs of NIDDM patients. Kidney Int. 2003;64(6): 2188-2195.

55. Marhoffer W, Stein M, Schleinkofer L, Federlin K. Monitoring of polymorphonuclear leukocyte functions in diabetes mellitus - a comparative study of conventional radiometric function tests and low-light imaging systems. J Biolumin Chemilumin. 1994;9(3):165-170.

56. Tennenberg SD, Finkenauer R, Dwivedi A. Absence of lipopolysaccharide-induced inhibition of neutrophil apoptosis in patients with diabetes. Arch Surg. 1999;134(11):1229-1233.

57. Kobayashi SD, Braughton KR, Palazzolo-Ballance AM, et al. Rapid neutrophil destruction following phagocytosis of Staphylococcus aureus. J Innate Immun. 2010;2(6):560-575.

58. Droemann D, Aries SP, Hansen F, et al. Decreased apoptosis and increased activation of alveolar neutrophils in bacterial pneumonia. Chest. 2000;117(6):1679-1684.

59. Wang CH, Yu CT, Lin HC, Liu CY, Kuo HP. Hypodense alveolar macrophages in patients with diabetes mellitus and active pulmonary tuberculosis. Tuber Lung Dis. 1999;79(4):235-242.

60. Andreasen AS, Pedersen-Skovsgaard T, Berg RM, et al. Type 2 diabetes mellitus is associated with impaired cytokine response and adhesion molecule expression in human endotoxemia. Intensive Care Med. 2010;36(9):1548-1555.

61. Restrepo BI, Fisher-Hoch SP, Pino PA, et al. Tuberculosis in poorly controlled type 2 diabetes: altered cytokine expression in peripheral white blood cells. Clin Infect Dis. 2008;47(5):634-641.

62. Martinez PJ, Mathews C, Actor JK, et al. Impaired CD4+ and T-helper 17 cell memory response to Streptococcus pneumoniae is associated with elevated glucose and percent glycated hemoglobin A1c in Mexican Americans with type 2 diabetes mellitus. Transl Res. 2014;163(1): $53-63$. 
63. Feuerer M, Herrero L, Cipolletta D, et al. Lean, but not obese, fat is enriched for a unique population of regulatory $\mathrm{T}$ cells that affect metabolic parameters. Nat Med. 2009;15(8):930-939.

64. Jagannathan-Bogdan M, McDonnell ME, Shin H, et al. Elevated proinflammatory cytokine production by a skewed $\mathrm{T}$ cell compartment requires monocytes and promotes inflammation in type 2 diabetes. J Immunol. 2011;186(2):1162-1172.

65. Restrepo BI, Fisher-Hoch SP, Smith B, Jeon S, Rahbar MH, McCormick JB. Mycobacterial clearance from sputum is delayed during the first phase of treatment in patients with diabetes. Am J Trop Med Hyg. 2008;79(4):541-544.

66. Tang S, Zhang Q, Yu J, et al. Extensively drug-resistant tuberculosis at a tuberculosis specialist hospital in Shanghai, China: clinical characteristics and treatment outcomes. Scand J Infect Dis. 2011;43(4): 280-285.

67. Jiménez-Corona ME, Cruz-Hervert LP, García-García L, et al. Association of diabetes and tuberculosis: impact on treatment and post-treatment outcomes. Thorax. 2013;68(3):214-220.
68. World Health Organization; Stop-TB Partnership. New Laboratory Diagnostic Tools for Tuberculosis Control. Geneva: World Health Organization; 2008. Available from: http://www.who.int/tdr/publications/disease/tuberculosis/en/. Accessed March 20, 2014.

69. American Diabetes Association. Standards of medical care in diabetes - 2010. Diabetes Care. 2010;33(Suppl 1):S11-S61.

70. Lenters-Westra E, Schindhelm RK, Bilo HJ, Slingerland RJ. Haemoglobin $\mathrm{A}_{1 \mathrm{c}}$ : Historical overview and current concepts. Diabetes Res Clin Pract. 2013;99(2):75-84.

71. Lin Y, Li L, Mi F, et al. Screening patients with diabetes mellitus for tuberculosis in China. Trop Med Int Health. 2012;17(10): 1302-1308.

72. Harries AD, Billo N, Kapur A. Links between diabetes mellitus and tuberculosis: should we integrate screening and care? Trans $R$ Soc Trop Med Hyg. 2009;103(1):1-2.

73. Kapur A, Harries AD. Cohort monitoring - as a tool to improve diabetes care services. Diabetes Res Clin Pract. 2013;102(3):260-264.
Research and Reports in Tropical Medicine

\section{Publish your work in this journal}

Research and Reports in Tropical Medicine is an international, peerreviewed, open access journal publishing original research, case reports, editorials, reviews and commentaries on all areas of tropical medicine, including: Diseases and medicine in tropical regions; Entomology; Epidemiology; Health economics issues; Infectious disease; Laboratory

\section{Dovepress}

science and new technology in tropical medicine; Parasitology; Public health medicine/health care policy in tropical regions; and Microbiology. The manuscript management system is completely online and includes a very quick and fair peer-review system. Visit http://www.dovepress com/testimonials.php to read real quotes from published authors. 